\title{
Galectin-3 facilitates the proliferation and migration of nasopharyngeal carcinoma cells via activation of the ERK1/2 and Akt signaling pathways, and is positively correlated with the inflammatory state of nasopharyngeal carcinoma
}

\author{
MEI LI ${ }^{1,2^{*}}$, YU BIN CHEN ${ }^{3 *}$, FEN LIU $^{1}$, JIA QUAN QU ${ }^{2}$, LI CHENG REN $^{4}$, JIN CHAI $^{2}$ and CAN E. TANG ${ }^{1,5}$ \\ ${ }^{1}$ Institute of Medical Science Research, Xiangya Hospital, Central South University, Changsha, Hunan 410008; \\ ${ }^{2}$ Cholestatic Liver Diseases Center and Department of Gastroenterology, Southwest Hospital, \\ Third Military Medical University, Chongqing 400038; ${ }^{3}$ Department of Cardiac Surgery, Xiangya Hospital, \\ Central South University, Changsha, Hunan 410008; Departments of ${ }^{4}$ Burn and Reconstructive Surgery and \\ ${ }^{5}$ Endocrinology, Xiangya Hospital, Central South University, Changsha, Hunan 410008, P.R. China
}

Received September 18, 2020; Accepted February 23, 2021

DOI: $10.3892 / \mathrm{mmr} .2021 .12009$

\begin{abstract}
Nasopharyngeal carcinoma (NPC) is an epithelial carcinoma originating from the nasopharyngeal mucosal tissue and is highly prevalent in southeast Asia. Galectin-3 (gal-3) serves crucial roles in many cancers but its role in NPC remains to be elucidated. The aim of the present study was to investigate the role of gal-3 in NPC. Immunohistochemistry and ELISA were used to determine the expression level of gal-3 in patients with NPC or chronic rhinitis (CR). Gal-3 short hairpin (sh)RNA was established to knockdown gal-3 in $5-8 \mathrm{~F}$ and $6-10 \mathrm{~B}$ cells, allowing for the evaluation of the roles of gal-3 in proliferation, migration and apoptosis in NPC cell lines. Immunohistochemistry staining of IL-6 and IL-8 was applied to access the inflammatory state of tumor tissues, and the correlation between the inflammatory state and gal-3 was analyzed. The results demonstrated that gal-3 was upregulated in patients with NPC compared with patients with CR. Knockdown of gal-3 inhibited proliferation and migration in $5-8 \mathrm{~F}$ and $6-10 \mathrm{~B}$ cells, as well as promoted apoptosis in these
\end{abstract}

Correspondence to: Professor Jin Chai, Cholestatic Liver Diseases Center and Department of Gastroenterology, Southwest Hospital, Third Military Medical University, 30 Yanzheng Street, Chongqing 400038, P.R. China

E-mail: jin.chai@cldcsw.org

Professor Can E. Tang, Institute of Medical Science Research, Xiangya Hospital, Central South University, 78 Xiangya Road, Changsha, Hunan 410008, P.R. China

E-mail: tangcane@csu.edu.cn

${ }^{*}$ Contributed equally

Key words: galectin-3, nasopharyngeal carcinoma, ERK1/2, Akt, inflammation cells. The expression levels of MMP-9 and IL- 8 were also decreased in 5-8F and 6-10B cells after transfection with gal-3 shRNA. A positive correlation was identified between the expression level of gal-3 and the inflammatory state of NPC. The phosphorylation levels of ERK1/2 and Akt were downregulated after knockdown of gal-3 in 5-8F and 6-10B cells. In conclusion, the expression level of gal-3 was upregulated in patients with NPC and was positively correlated with the inflammatory state of NPC. The results suggested that gal-3 promoted the proliferation and migration of 5-8F and 6-10B cells, while inhibiting the apoptosis of these cells. Moreover, activation of ERK1/2 and Akt may be the underlying mechanism of the effects of gal-3 on NPC.

\section{Introduction}

Nasopharyngeal carcinoma (NPC), an epithelial carcinoma originating from the nasopharyngeal mucosal tissue, is widely distributed in Southeast Asia, and especially in southern China (1-3). The incidence of NPC in southern China is 60 per 100,000 individuals, and the corresponding mortality in 2015 was 34 per 100,000 individuals (4,5). Metastasis usually occurs in the early stage of NPC and is the main cause of patient mortality (6). Therefore, it is urgent for researchers to examine the underlying mechanism of the development and metastasis of NPC and to identify potential therapeutic targets for NPC.

Galectin-3 (gal-3), a member of the $\beta$-galactoside-binding protein family, is characterized by a specific chimeric structure containing a unique carbohydrate recognition domain and multifunctional N-terminal domain (7). Gal-3 is involved in cellular homeostasis, organogenesis, angiogenesis, tumor invasion and metastasis (8). Research has indicated that the upregulation of gal-3 promotes neoplastic transformation and contributes to the phenotype maintenance of malignant breast cells $(9,10)$. In addition, patients with tumor metastasis exhibited significantly higher concentrations of circulating 
gal-3 compared with healthy individuals (11). Gal-3 was also found to induce the formation of new capillaries in vivo and sustain the angiogenic capability of tumor-associated macrophages $(12,13)$. Thus, it was hypothesized that gal-3 may be associated in the development and metastasis of NPC. ERK1/2 and Akt signaling pathways are crucial regulators in the development and metastasis of NPC $(14,15)$. However, whether gal-3 is involved in the activation of ERK1/2 and Akt signaling pathways remains unknown and requires further investigation.

In the present study, tumor tissue samples from patients with NPC and nasopharyngeal tissues from patients with chronic rhinitis (CR) were collected, and the difference in the expression levels of gal-3 was investigated. Subsequently, the NPC cell lines, 5-8F and 6-10B, and the nasopharyngeal epithelium cell line, NP69, were used to examine the function and potential mechanism of gal-3 in NPC.

\section{Materials and methods}

Collection of human samples. The study was approved by the Ethics Committee of Xiangya Hospital Central South University. In total, 40 tumor specimens and 37 serum samples $(5 \mathrm{ml})$ were obtained from 40 patients with NPC, and 15 nasopharyngeal tissues and 26 serum samples were collected from 26 patients with CR in Xiangya Hospital Central South University between January 2015 and January 2018. Written informed consent was obtained from all patients prior to inclusion in the study. Characteristics of the patients, including sex, age and TNM stage, were recorded. The tissues were fixed in $4 \%$ formalin at room temperature for $24 \mathrm{~h}$ and embedded in paraffin for subsequent histological experiments, and the serum was stored at $-80^{\circ} \mathrm{C}$.

Cell culture. The NPC cell line, 5-8F, was kindly provided by the Cancer Research Institute of Central South University. The NPC cell line, 6-10B, and the immortalized nasopharyngeal epithelium cell line, NP69, were purchased from Hunan Fenghui Biotechnology Co., Ltd. 5-8F and 6-10B cells were cultured in high-glucose DMEM (Gibco; Thermo Fisher Scientific, Inc.) supplemented with 10\% FBS (Gibco; Thermo Fisher Scientific, Inc.) and 1\% penicillin-streptomycin. NP69 cells were cultured in keratinocyte serum-free medium (Invitrogen; Thermo Fisher Scientific, Inc.). All cells were cultured in a $37^{\circ} \mathrm{C}$ incubator with $5 \% \mathrm{CO}_{2}$.

Gal-3 short hairpin (sh)RNA (1,000 ng/ $\mu \mathrm{l})$ and control shRNA $(1,000 \mathrm{ng} / \mu \mathrm{l})$ (Shanghai Genechem Co., Ltd.) were transfected into cells at room temperature for $15 \mathrm{~min}$ using the FuGENE HD transfection reagent (Promega Corporation), according to the manufacturer's recommendation. After a month and a half of screening, subsequent experiments were performed. The sequences of the gal-3 shRNA and control shRNA are presented in Tables SI and SII.

Reverse transcription-quantitative PCR (RT-qPCR). Total RNA was isolated using RNAiso Plus reagent (Takara Bio, Inc.) and reverse transcribed using the PrimeScript RT Reagent kit with gDNA Eraser (Takara Bio, Inc.). The temperature and duration of reverse transcription was: $37^{\circ} \mathrm{C}$ for $15 \mathrm{~min}, 85^{\circ} \mathrm{C}$ for $5 \mathrm{sec}$. Relative gene expression levels were measured using SYBR Premix Ex Taq II (Takara Bio, Inc.). The thermocycling conditions were: Holding stage, $95^{\circ} \mathrm{C}$ for $30 \mathrm{sec}$; PCR stage, $95^{\circ} \mathrm{C}$ for $5 \mathrm{sec}, 58^{\circ} \mathrm{C}$ for $30 \mathrm{sec}, 40$ cycles; melt curve stage, $95^{\circ} \mathrm{C}$ for $15 \mathrm{sec}, 60^{\circ} \mathrm{C}$ for $60 \mathrm{sec}$ and $95^{\circ} \mathrm{C}$ for $15 \mathrm{sec}$. The results were quantitatively analyzed according to the $2^{-\Delta \Delta C q}$ method (16). The sequences of the primers used for RT-qPCR analysis are presented in Table SIII.

Immunoblotting. For immunoblotting, whole cell lysates were prepared in RIPA buffer (Sigma-Aldrich; Merck KGaA) containing protease inhibitor and phosphatase inhibitor (Roche Diagnostics). Protein concentration was determined using a BCA protein assay kit (Thermo Fisher Scientific, Inc.). Then, protein samples $(20 \mu \mathrm{g})$ were separated by SDS-PAGE and subsequently transferred to PVDF membranes (EMD Millipore). The membranes were blocked in 5\% milk for $1 \mathrm{~h}$ at $37^{\circ} \mathrm{C}$ before incubation with primary mouse monoclonal anti-gal 3 antibody (1:1,000; cat. no. 60207-1-lg; ProteinTech Group, Inc.), rabbit monoclonal anti-phosphorylated (p)-Akt (S473) antibody (1:1,000; cat. no. BS9913M; Bioworld Technology, Inc.), rabbit polyclonal anti-Akt antibody (1:1,000; cat. no. BS1810; Bioworld Technology, Inc.), rabbit monoclonal anti-p-ERK1/2 antibody (1:1,000; cat. no. 4370; Cell Signaling Technology, Inc.), rabbit monoclonal anti-ERK1/2 antibody (1:1,000; cat. no. 4695; Cell Signaling Technology, Inc.) and rabbit polyclonal anti-GAPDH antibody (1:5,000; cat. no. 10494-1-AP; ProteinTech Group, Inc.) overnight at $4{ }^{\circ} \mathrm{C}$. Then, the membranes were incubated with HRP-coupled secondary antibodies (Goat Anti-Rabbit IgG(H+L), HRP conjugate, 1:10,000; cat. no. SA00001-2 and Goat Anti-mouse $\operatorname{IgG}(\mathrm{H}+\mathrm{L})$, HRP conjugate, 1:10,000; cat. no. SA00001-2; ProteinTech Group, Inc.) for $1 \mathrm{~h}$ at $37^{\circ} \mathrm{C}$. After washing with PBS, the membranes were visualized using ECL reagent (cat. no. p10100; NCM Biotech) and imaged using X-ray film. ImageJ software was used for densitometry (version number: $1.51 \mathrm{v}$, https://imagej.net/downloads).

ELISA. The concentration of gal-3 in serum was determined using the Human Galectin-3 ELISA kit (cat. no. E-EL-H1470c; Elabscience, Inc.) according to the manufacturer's recommendation.

Immunohistochemical assay. Paraffin-embedded sections (tissues fixed in 4\% formalin at room temperature for $24 \mathrm{~h}$; section thickness, $4 \mu \mathrm{m}$ ) were deparaffinized and hydrated $(100,95,80$ and $70 \%)$. After antigen retrieval, sections were blocked using normal goat serum (cat. no. ZLI-9022; OriGene Technologies, Inc.) at room temperature for $30 \mathrm{~min}$ and then were incubated with primary antibodies against gal-3 (1:500; cat. no. 60207-1-lg; ProteinTech Group, Inc.), IL-6 (1:200, cat. no. ab6672; Abcam) and IL-8 (1:400; cat. no. 27095-1-AP; ProteinTech Group, Inc.). Then, sections were stained using a detection kit (cat. no. PV9000; OriGene Technologies, Inc.) at room temperature for $60 \mathrm{sec}$ and hematoxylin at room temperature for $30 \mathrm{sec}$ (both from OriGene Technologies, Inc.). Images (10 fields for each section) were obtained using light microscope with a digital camera (Olympus Corporation) at $x 400$ magnification. According to the method described by Hara and Okayasu (17), the present study blindly analyzed the expression levels of gal-3, IL- 6 and IL- 8 in tissues. The immunohistochemistry score was determined as follows: 
Staining percentage: No positive cells, $0 ; \leq 25 \%$ positive cells, $1 ; 26-50 \%$ positive cells, 2 ; $51-75 \%$ positive cells, 3 ; and $>75 \%$ positive cells, 4 ; and staining intensity: No staining, 0 ; weak staining, 1; moderate staining, 2; and dark staining, 3 . Comprehensive score $=$ staining percentage + staining intensity. Comprehensive scores of gal-3, IL- 6 and IL- $8 \geq 4$ were classified as high expression; scores of $<4$ were classified as low expression.

Cell proliferation . 5-8F and 6-10B cells were seeded in 96-well plates at a density of 4,000 cells per well. After $24 \mathrm{~h}, 20 \mu \mathrm{l}$ MTT solution was added. Then, $4 \mathrm{~h}$ later, MTT was removed, $100 \mu 1$ DMSO was added and the absorbance at $490 \mathrm{~nm}$ was measured (Varioskan Flash; Thermo Scientific, Inc.).

Hoechst staining. 5-8F and 6-10B cells $\left(1 \times 10^{3}\right)$ were cultured in 96-well plates and incubated at $37^{\circ} \mathrm{C}$ with $5 \% \mathrm{CO}_{2}$ for $24 \mathrm{~h}$. The cells were washed twice with PBS and incubated at room temperature with glacial acetic acid/methanol mixture (glacial acetic acid: Methanol=1:3) for $30 \mathrm{~min}$. After washing with PBS, the cells were incubated with $1 \mu \mathrm{g} / \mathrm{ml}$ Hoechst 33258 solution for $10 \mathrm{~min}$ in the dark at $37^{\circ} \mathrm{C}$. Images were then acquired using a fluorescent PerkinElmer Operetta CLS system (PerkinElmer, Inc.) at x400 magnification; six fields were randomly chosen.

5-Ethynyl-2'-deoxyuridine (Edu) labeling Assay. 5-8F and $6-10 \mathrm{~B}$ cells $\left(1 \times 10^{3}\right)$ were cultured in 96 -well plates and incubated at $37^{\circ} \mathrm{C}$ with $5 \% \mathrm{CO}_{2}$ for $24 \mathrm{~h}$. Cells were incubated with EdU solution (50 $\mu \mathrm{M}$; B8010; Beijing Solarbio Science \& Technology Co., Ltd.) for $2 \mathrm{~h}$ at room temperature and then was fixed using $4 \%$ paraformaldehyde at room temperature for $30 \mathrm{~min}$. Cells were incubated with glycine (50 $\mu \mathrm{l} ; 2 \mathrm{mg} / \mathrm{ml}$; cat. no. ST085; Beyotime Institute of Biotechnology) for $5 \mathrm{~min}$ at room temperature and washed with PBS thrice. After that, cells were permeated with $0.5 \%$ Triton X-100 (cat. no. ST797; Beyotime Institute of Biotechnology) for $10 \mathrm{~min}$ at room temperature and stained with Apollo staining solution (cat. no. CA1170; Beijing Solarbio Science \& Technology Co., Ltd.) for $30 \mathrm{~min}$ at room temperature. Subsequently, cells were washed with PBS thrice and stained with Hoechst 33342 solution (cat. no. B8040; Beijing Solarbio Science \& Technology Co., Ltd.) for $30 \mathrm{~min}$ at room temperature. Images were then acquired using a fluorescent PerkinElmer Operetta CLS system (PerkinElmer, Inc.) at X400 magnification; six fields were randomly chosen.

Scratch assay. 5-8F and 6-10B cells were seeded in 6-well plates at a density of $2 \times 10^{5} / \mathrm{ml}$ and incubated at $37^{\circ} \mathrm{C}$ with $5 \% \mathrm{CO}_{2}$. For the scratch assay, cells were scratched using $10-\mu 1$ pipette tips. During cultivation, the medium was replaced with high-glucose DMEM containing 1\% FBS. Images of cells were taken at x100 magnification after 0 , 24 and 48 h (Olympus Corporation). The wound closure rate [(initial wound area-wound area at $24 \mathrm{~h}$ )/initial wound area] was analyzed using Image Pro Plus 6.0 software (Media Cybernetics, Inc.) (18).

Migration assay. Cells were collected and resuspended in serum-free DMEM. Then, $\sim 5 \times 10^{4}$ cells in $200 \mu 1$ serum-free
DMEM were seeded in the upper chamber, while the lower chamber was filled with $600 \mu 1$ DMEM containing $15 \%$ FBS. After $24 \mathrm{~h}$ incubation at $37^{\circ} \mathrm{C}$ with $5 \% \mathrm{CO}_{2}$, the cells on the upper face were wiped off, while those that migrated to the lower face were fixed using $4 \%$ paraformaldehyde at room temperature for $20 \mathrm{~min}$ and stained with crystal violet solution (cat. no. C0121; Beyotime Institute of Biotechnology) for 5 min. Images were obtained using a microscope (Olympus Corporation). The number of migrated cells was counted in three random fields (x200 magnification).

Statistical analysis. All experiments were repeated three times. All analyses were performed using SPSS 18.0 (SPSS, Inc.) and GraphPad Prism 8 (GraphPad Software, Inc.). The numerical data are presented as the mean \pm SEM, and were analyzed using an unpaired Student's t-test or one-way ANOVA followed by Tukey's multiple comparisons test post hoc test. The Fisher's exact test and Pearson's correlation were used to analyze the difference in the expression levels of gal-3 and the relationship between gal-3 and inflammation cytokines. $\mathrm{P}<0.05$ was considered to indicate a statistically significant difference.

\section{Results}

Expression levels of gal-3 are upregulated in patients with NPC and in NPC cell lines. The expression levels of gal-3 in tumor tissues and nasopharyngeal tissues were determined via immunochemistry. The results demonstrated that gal-3 was mainly expressed in the cytoplasm and was significantly upregulated in patients with NPC compared with patients with CR (Fig. 1A; Table I). Furthermore, the results of the ELISA showed that the concentration of circulating gal-3 in patients with NPC was significantly higher compared with that of patients with CR (Fig. 1B). The mRNA expression levels of gal-3 in the NPC cell lines, 5-8F and 6-10B, were significantly higher compared with those of NP69, a nasopharyngeal epithelium cell line (Fig. 1C). In addition, immunoblotting demonstrated that the expression levels of gal-3 protein in 5-8F and 6-10B cells were significantly upregulated compared with those of NP69 cells (Fig. 1D). However, there was no correlation between the expression levels of gal-3 in tumor tissues and the TNM stage of patients with NPC (Table SIV).

Knockdown of gal-3 inhibits the proliferation and migration of NPC cell cells and induces their apoptosis. Transfection of gal-3 shRNA was capable of downregulating the protein and mRNA expression levels of gal-3 in 5-8F and 6-10B cells (Fig. 2A and B). The MTT proliferation assay demonstrated that, after transfection with gal-3 shRNA, 5-8F and 6-10B cells exhibited significantly impaired proliferative capabilities compared with cells transfected with control shRNA (Fig. 2C). The results of EdU staining indicated that knockdown of gal-3 significantly inhibited the proliferation of 5-8F and 6-10B cells, compared with cells transfected with control shRNA, while the results of Hoechst 33258 staining suggested that knockdown of gal-3 promoted apoptosis in 5-8F and 6-10B cells (Fig. 2D and E). On the other hand, based on the scratch assay used to determine the mobility of $5-8 \mathrm{~F}$ and 6-10B cells, knockdown of gal-3 in NPC cells significantly 
A

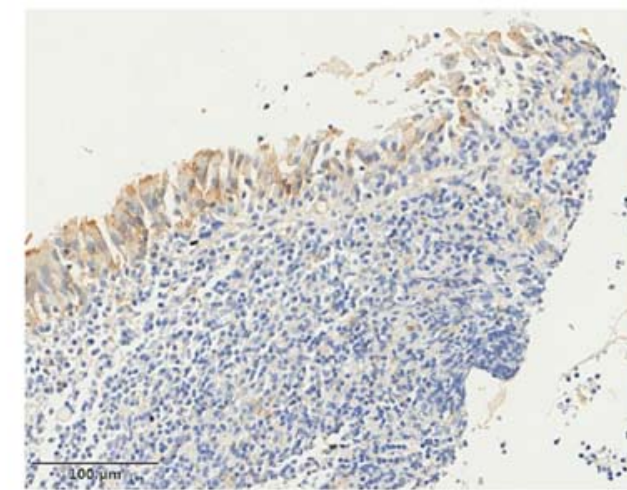

CR
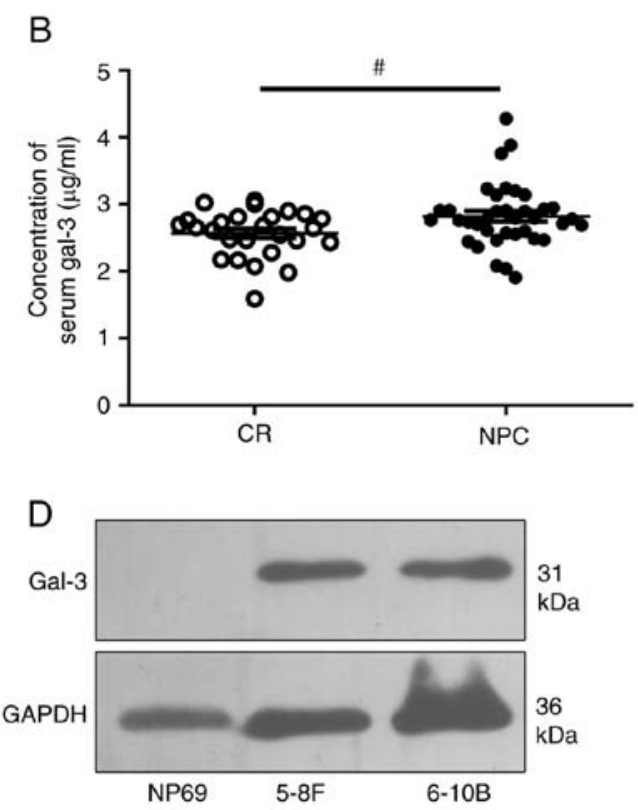

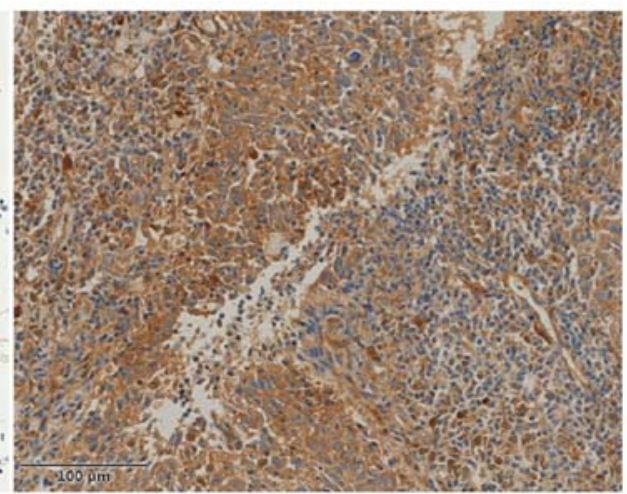

NPC
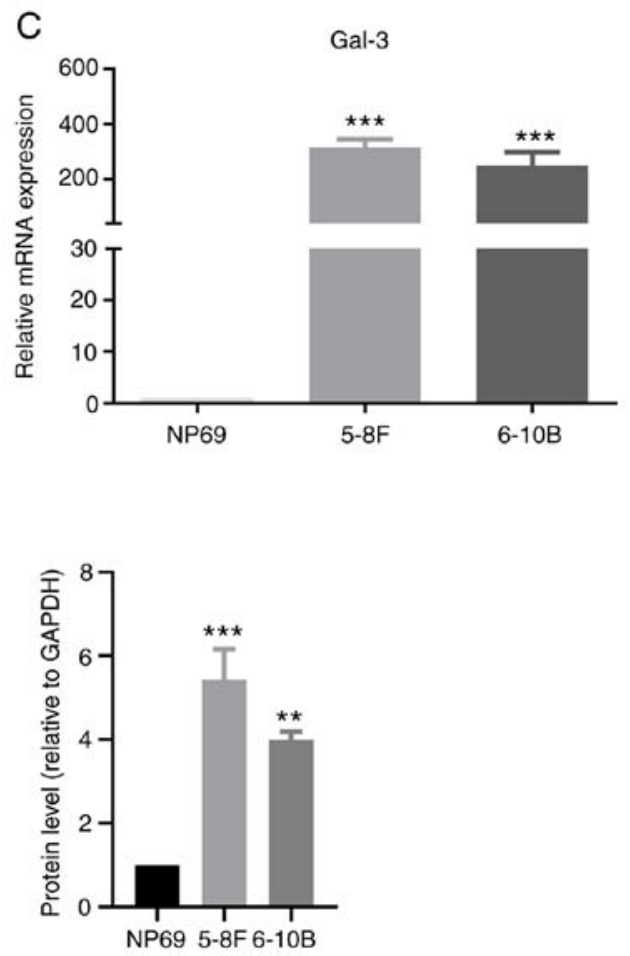

Figure 1. Expression levels of gal-3 are upregulated in patients with NPC and in NPC cell lines. (A) Representative images of immunochemistry staining of gal-3 in nasopharyngeal tissues and tumor tissues at x400 magnification. (B) Concentrations of serum gal-3 in patients with NPC and patients with CR were determined via ELISA. ${ }^{\text {P }}<0.05$. (C) mRNA expression levels of gal-3 in NP69, 5-8F and 6-10B cells were detected using reverse transcription-quantitative PCR and normalized to GAPDH. (D) Protein expression levels of gal-3 in NP69, 5-8F and 6-10B cells were detected via western blotting, using GAPDH as the loading control. ${ }^{* *} \mathrm{P}<0.01,{ }^{* * *} \mathrm{P}<0.001$ vs. NP69 cells. Gal-3, galectin-3; NPC, nasopharyngeal carcinoma; CR, chronic rhinitis.

decreased their migratory capability compared with the results of cells transfected with control shRNA (Fig. 3A and B). A Transwell migration assay further validated these findings (Fig. 3C and D). In addition, the mRNA expression levels of MMP-9 in 5-8F and 6-10B cells were significantly downregulated after transfection with gal-3 shRNA (Fig. 3E).

Gal-3 is associated with the inflammatory state of NPC. The expression levels of the inflammatory cytokines, IL- 6 and IL-8, were measured using a immunohistochemical assay to assess the inflammatory state of the tumor tissue. The results suggested that IL-6 and IL-8 were mainly expressed in the cytoplasm and extracellular matrix. The relationship between gal-3 and the inflammatory cytokines was analyzed, and the results demonstrated that the expression level of gal-3 was positively correlated with the expression levels of IL-6 and IL-8, which suggests that gal-3 was associated with the inflammatory state of NPC (Fig. 4A). Furthermore, 5-8F and 6-10B cells transfected with gal-3 shRNA exhibited significantly lower expression levels of IL-8 compared with cells transfected with control shRNA (Fig. 4B).

Knockdown of gal-3 suppresses the ERK1/2 and Akt signaling pathways. The ERK1/2 and Akt signaling pathways are involved in the processes of tumor survival and migration (19). After transfection with gal-3 shRNA, the phosphorylation levels of ERK1/2 and Akt were significantly decreased in 5-8F and 6-10B cells, compared with cells transfected with control shRNA (Fig. 5A and B).

\section{Discussion}

Compared with other types of cancer, NPC is relatively rare, accounting for only $0.7 \%$ of all cancer types in 2018 (20). 
Table I. Expression levels of gal-3 in patients with NPC compared with patients with CR.

Expression levels of gal-3

\begin{tabular}{lcccc} 
Group & Cases (n) & High expression & Low expression & P-value \\
\hline CR & 15 & 7 & 8 & 0.0015 \\
NPC & 40 & 36 & 4 &
\end{tabular}

The Fisher's exact test was used to analyze the expression levels of gal-3 in patients with NPC and patients with CR. CR, chronic rhinitis; NPC, nasopharyngeal carcinoma; Gal-3, galectin-3.

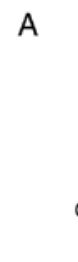

B
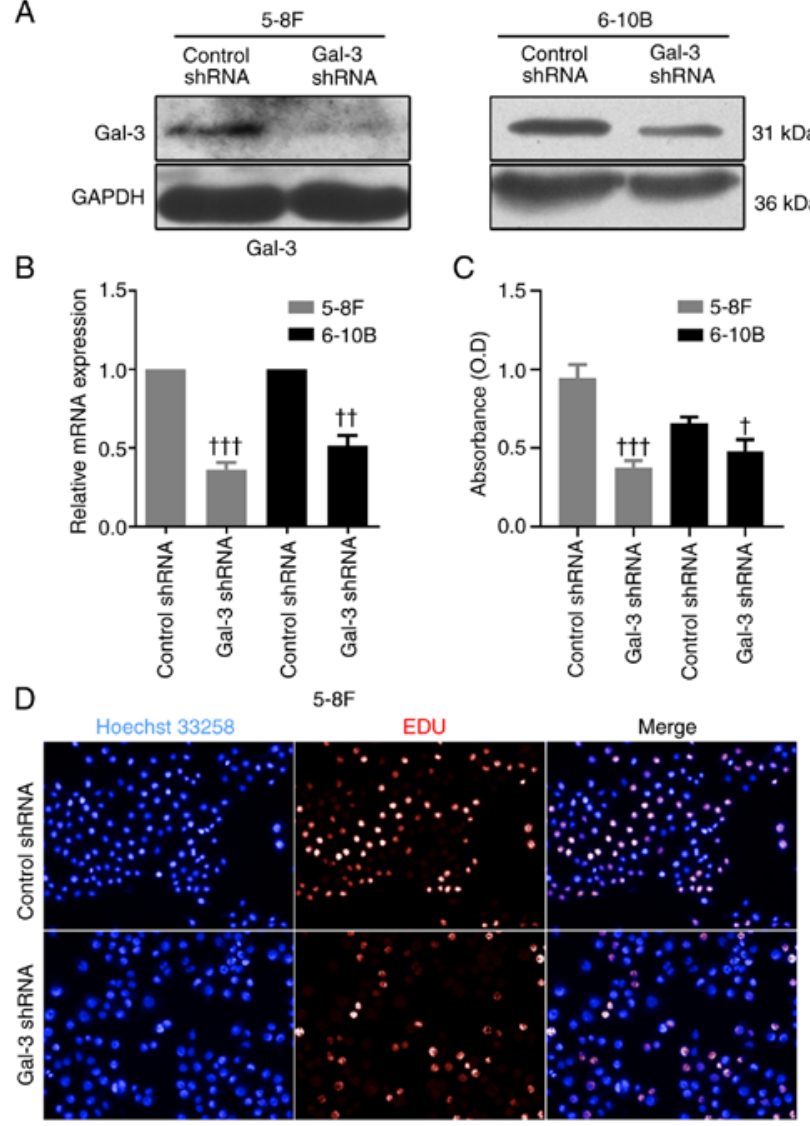

E

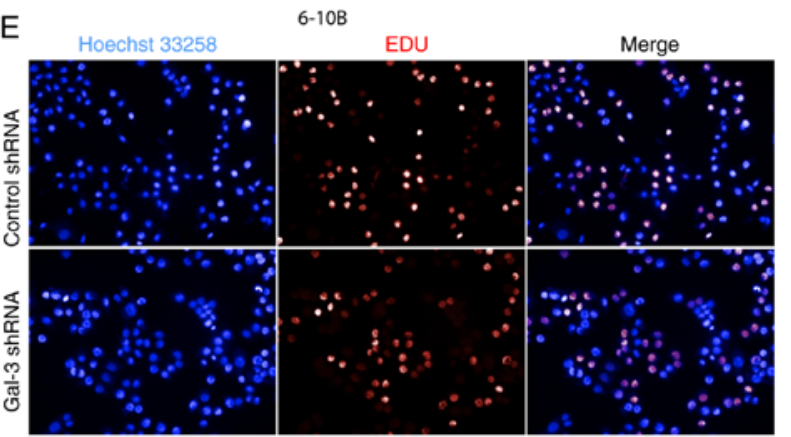

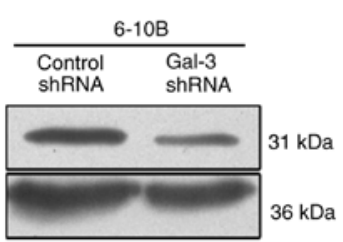

C

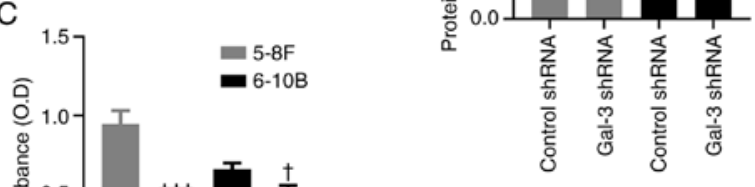

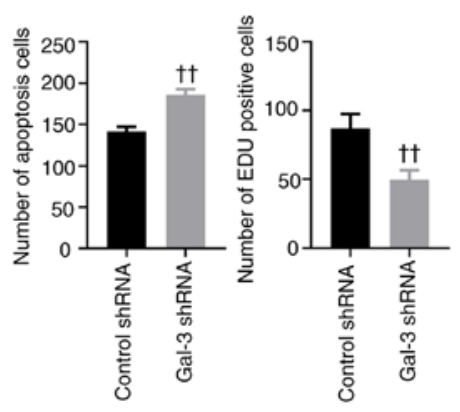

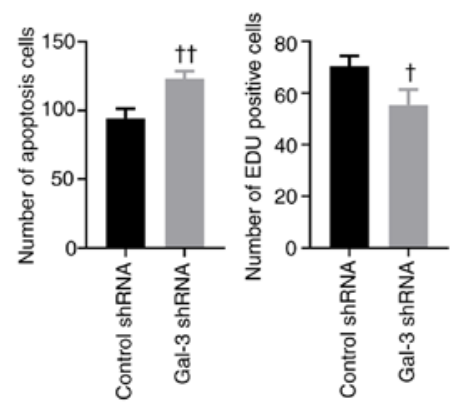

Figure 2. Knockdown of gal-3 inhibits the proliferation of 5-8F and 6-10B cells, while inducing their apoptosis. (A) Protein expression levels of gal-3 in 5-8F and 6-10B cells transfected with control shRNA or gal-3 shRNA were determined via western blotting, using GAPDH as the loading control. (B) mRNA expression levels of gal-3 in 5-8F and 6-10B cells transfected with control shRNA or gal-3 shRNA were determined via reverse transcription-quantitative PCR. (C) A MTT proliferation assay using was used to investigate the proliferation of 5-8F and 6-10B cells transfected with control shRNA or gal-3 shRNA. Representative images of Hoechst 33258 and EdU staining of (D) 5-8F and (E) 6-10B cells transfected with control shRNA or gal-3 shRNA to show their apoptosis and proliferation (magnification, $\mathrm{x} 400$ ). ${ }^{\dagger} \mathrm{P}<0.05,{ }^{\dagger} \mathrm{P}<0.01,{ }^{\dagger \dagger} \mathrm{P}<0.001$ vs. control shRNA. shRNA, short hairpin RNA; Gal-3, galectin-3; EdU, 5-Ethynyl-2'-deoxyuridine; O.D, optical density. 

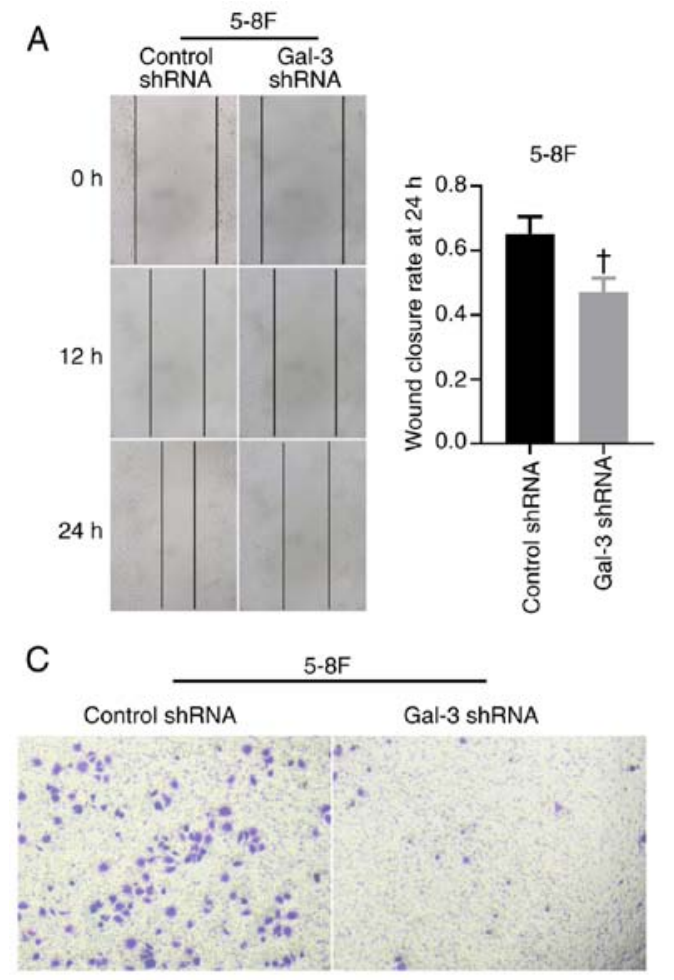

$\mathrm{D}$

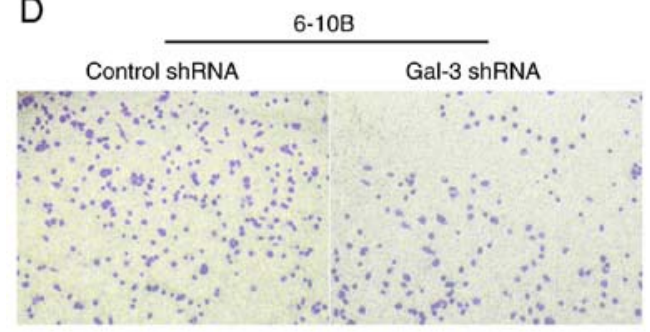

B
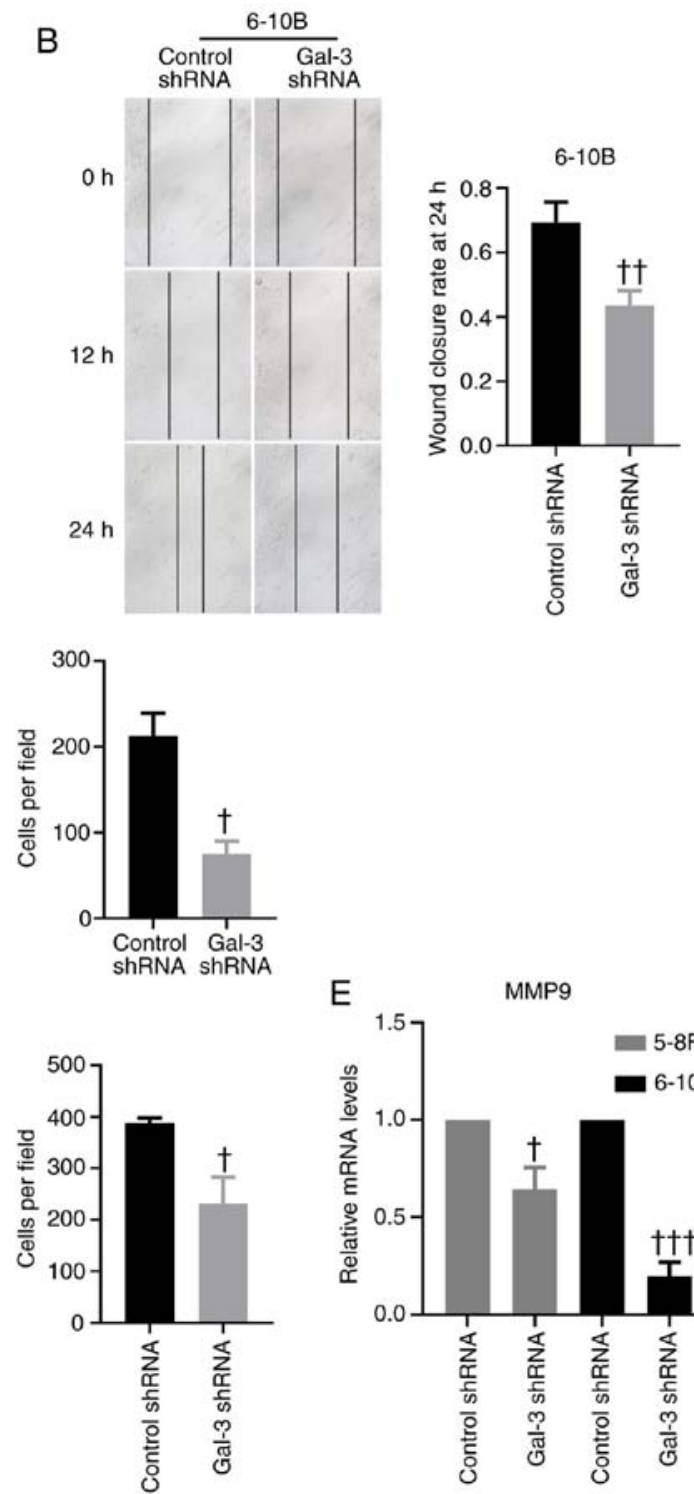

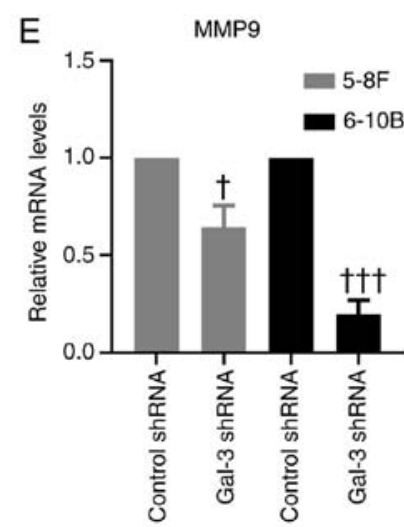

Figure 3. Knockdown of gal-3 inhibits the migration of 5-8F and 6-10B cells. Representative images of the scratch assay demonstrating the mobility of (A) 5-8F and (B) 6-10B transfected with control shRNA or gal-3 shRNA (magnification, x100). Representative images of a Transwell assay showing the capability of migration of (C) 5-8F and (D) 6-10B transfected with control shRNA or gal-3 shRNA (magnification, x200). (E) mRNA expression levels of MMP-9 in $5-8 \mathrm{~F}$ and $6-10 \mathrm{~B}$ cells transfected with control shRNA or gal-3 shRNA were investigated via reverse transcription-quantitative $\mathrm{PCR}$. ${ }^{\dagger} \mathrm{P}<0.05,{ }^{\dagger} \mathrm{P}<0.01$, P $<0.001$ vs. control shRNA. shRNA, short hairpin RNA; Gal-3, galectin-3.

However, $>70 \%$ of new NPC cases are diagnosed in Southeast Asia, indicating an extremely unbalanced global distribution (20). In addition, NPC is one of the most aggressive cancer types and is characterized by frequent lymph node metastasis (21). Therefore, most patients with NPC are diagnosed at the advanced stage (22). Radiotherapy and chemotherapy are the conventional treatments for NPC, but the prognosis for patients at the advanced stage remains poor (23). Under such circumstances, it is important to investigate the underlying mechanism of occurrence and development of NPC.

Gals are carbohydrate-binding proteins, and members of the galectin family serve important roles in different types of cancer (24). Using proteomic analysis, our previous study reported that gas-1 acted as a biomarker for NPC (25). Gal-3 exhibits a specific chimeric structure, and is associated with the development of tumors and metastasis (8). Gal-3 is also significantly upregulated in Caco2 and DLD-1 cells, which are both human colon cancer cell lines, and the inhibition of gal-3 can suppress the mobility of these cells (26). Under hypoxic conditions, the secretion of gal-3 is significantly upregulated in tumor-associated macrophages, and the upregulation of gal-3 can result in the progression of breast cancer (27). Moreover, forkhead box D1 and gal-3 form a positive regulatory loop to modulate the aggressiveness of lung cancer (28). In head and neck adenoid cystic carcinoma, gal-3 was found to have a close relationship with distant metastasis (29). Thus, it was suggested that gal-3 may be associated with the development and metastasis of NPC.

In the present study, tumor tissues and nasopharyngeal tissues were obtained from patients with NPC and CR, respectively, and the immunochemistry results indicated that gal-3 was significantly upregulated in the tumor tissues from patients with NPC compared with the nasopharyngeal tissues from patients with CR. Furthermore, the concentration of circulating gal-3 in patients with NPC was significantly higher compared with that 

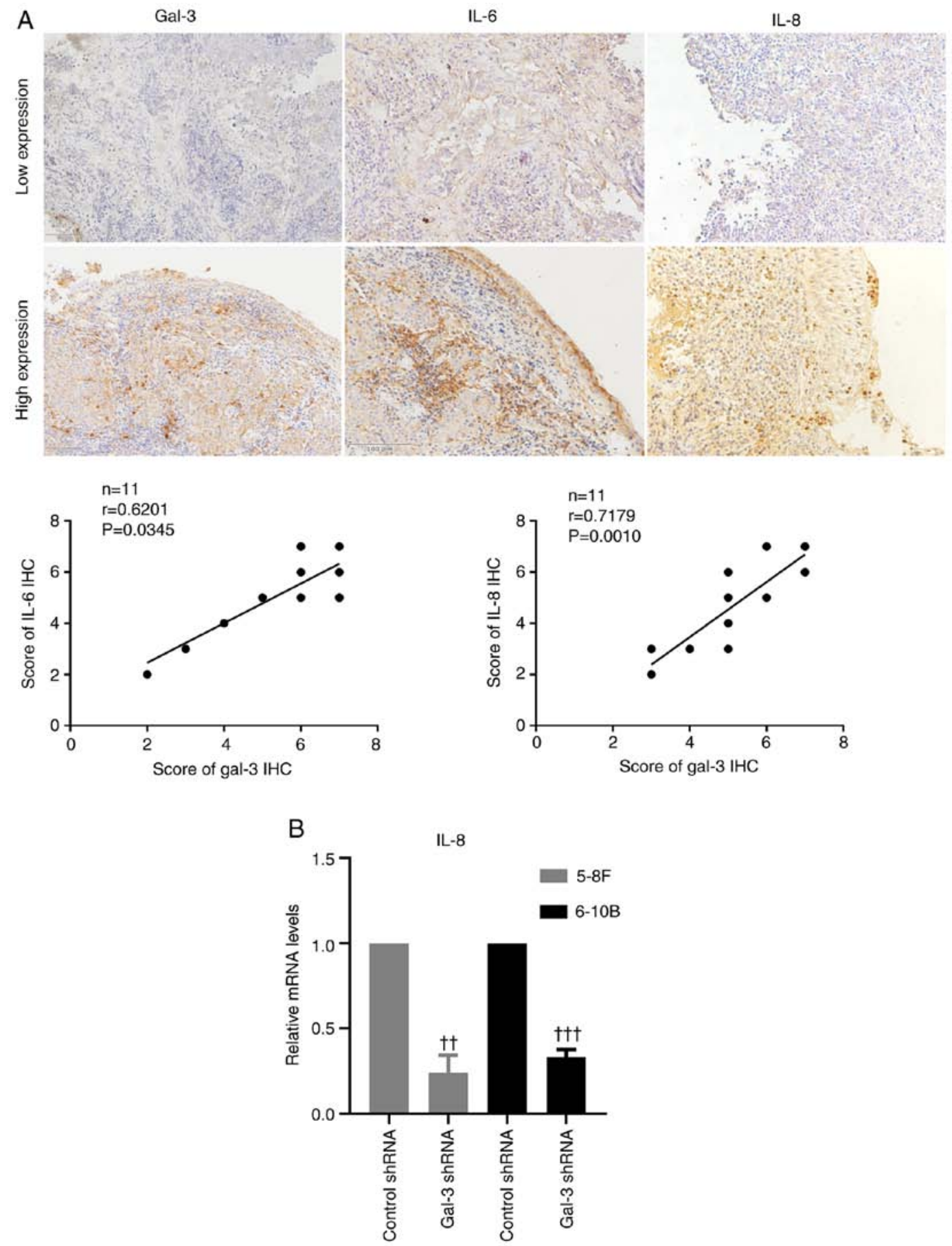

Figure 4. Gal-3 is associated with the inflammatory state of NPC. (A) Representative images of IHC staining gal-3, IL-6 and IL-8 in tumor tissues (magnification, $\mathrm{x} 400$ ). The Spearman's correlation analyzation indicated that the expression level of gal-3 was positively correlated with the expression levels of IL- 6 and IL-8 in tumor tissues. (B) mRNA expression levels of IL-8 in 5-8F and 6-10B cells transfected with control shRNA or gal-3 shRNA were determined using reverse transcription-quantitative PCR. ${ }^{\dagger} \mathrm{P}<0.01,{ }^{\dagger \dagger} \mathrm{P}<0.001$ vs. control shRNA. shRNA, short hairpin RNA; Gal-3, galectin-3; IHC, immunohistochemistry.

of patients with CR. These results implied that gal-3 may be involved in the development and metastasis of NPC. The NPC cell lines, 5-8F and 6-10B, and immortalized nasopharyngeal epithelium cell line NP69 were also used to examine the role of gal-3 in NPC. Consistent with the expression level of gal-3 in vivo, 5-8F and 6-10B cells exhibited significantly greater expression levels of gal-3 compared with NP69.

In the subsequent experiment, gal-3 shRNA was established to investigate the roles of gal-3 in NPC cells. After transfection with gal-3 shRNA, the proliferation and migration of 5-8F and 6-10B cells were significantly inhibited, while apoptosis in 5-8F and 6-10B cells was significantly activated. In addition, the expression level of MMP-9 was upregulated in cells transfected with gal-3 shRNA. MMPs are involved in the modulation of cell-cell interaction, cell-matrix interaction and extracellular matrix remodeling, and are associated with tumor metastasis (30). MMP-9 acts as a crucial regulator of metastasis of NPC and may be a potential therapeutic target for patients with NPC $(31,32)$. The findings of the present study suggested that gal-3 served important roles in the proliferation, 
A

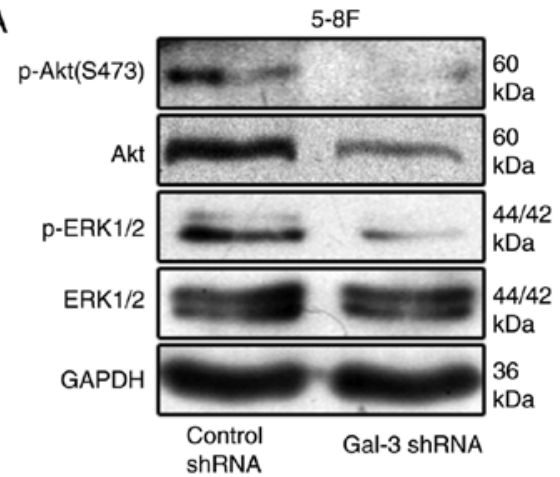

$B$

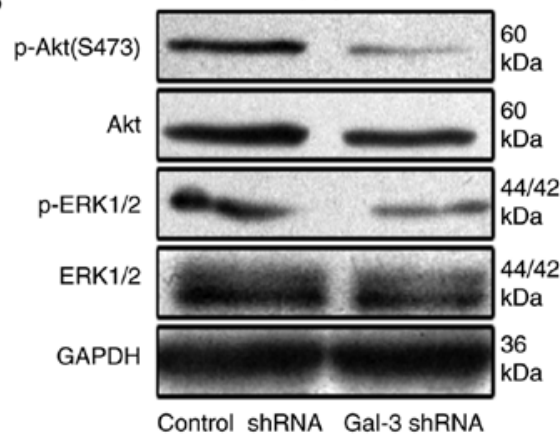

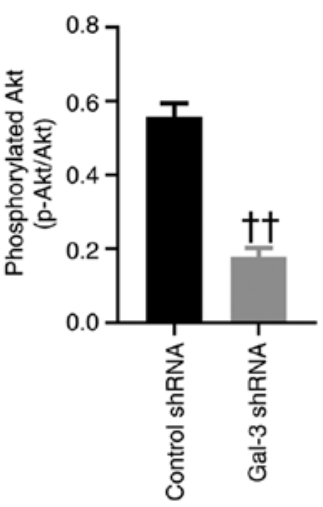
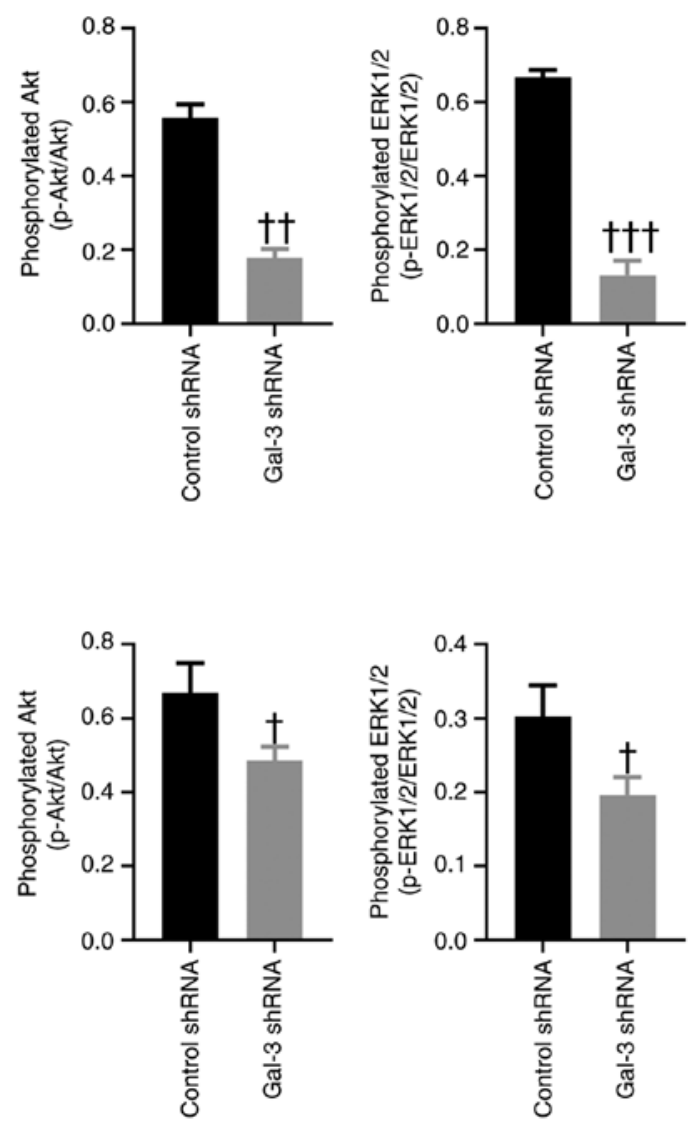

Figure 5. Knockdown of gal-3 suppresses the ERK1/2 and Akt signaling pathways. Phosphorylation levels of ERK1/2 and Akt in (A) 5-8F and (B) 6-10B cells transfected with control shRNA or gal-3 shRNA were detected via western blotting, using GAPDH as the loading control. ${ }^{\dagger}<0.05,{ }^{\dagger \dagger} \mathrm{P}<0.01,{ }^{\dagger \dagger} \mathrm{P}<0.001$ vs. control shRNA. shRNA, short hairpin RNA; Gal-3, galectin-3; p-, phosphorylated.

migration and apoptosis of NPC cells, and a regulate the development and metastasis of NPC in vivo. However, analysis of the correlation between the expression level of gal-3 and the TNM stage of patients with NPC suggested that there was no significant correlation between the two (Table SIV). This may be due to the relatively small number of cases in the present study (40 cases). Another explanation may be that the majority of the patients were at TNM stage III-IV (Table SIV).

The NF- $\mathrm{kB}$ signaling pathway regulates the development of NPC, and the dysregulation of NF- $\mathrm{KB}$ is regarded as a vital component of NPC tumorigenesis (33). NF- $\mathrm{KB}$ mediates the inflammatory response inside tumors and leads to the accumulation of proinflammatory cytokines in tumor tissues, which contributes to the tumor microenvironment, and eventually, tumorigenesis (34). ILs are multifunctional inflammatory cytokines that can regulate inflammatory responses and are considered important oncogenic mediators (35). Gal-3 is known to provoke an inflammatory reaction in acute inflammatory diseases, such as pneumonia, while inducing wound healing and fibrosis in chronic inflammatory diseases $(36,37)$. The role of gal-3 in the inflammation state of NPC, however, requires further investigation. In the present study, the expression levels of IL-6 and IL-8 in tumor tissue samples from patients with NPC were determined using immunohistochemistry to assess the inflammatory state of these tissues. It was found that the expression level of gal-3 in tumor tissues was positively correlated with the expression levels of IL-6 and
IL-8 in tumor tissues. Furthermore, after transfection with gal-3 shRNA, the expression level of IL-8 in 5-8F and 6-10B cells was significantly downregulated compared with that of cells transfected with control shRNA. These results suggested that gal-3 may be involved in the inflammatory response of NPC.

Finally, the current study sought to define the underlying mechanism of the effect of gal-3 on NPC cells. The activation of the ERK1/2 and Akt signaling pathways is involved in the proliferation, migration, invasion, metastasis and radiosensitivity of NPC (38-40). However, whether gal-3 affects the ERK1/2 and Akt signaling pathways of NPC cell lines remains unknown. Thus, in the present study, the phosphorylation levels of ERK1/2 and Akt were investigated in 5-8F and 6-10B cells transfected with gal-3 shRNA or control shRNA, and the results of immunoblotting indicated that gal-3 knockdown leads to the suppression of the ERK1/2 and Akt signaling pathways in $5-8 \mathrm{~F}$ and $6-10 \mathrm{~B}$ cells. These results suggest that activation of the ERK1/2 and Akt signaling pathways may be the potential mechanism of the effect of gal-3 on NPC cells.

In conclusion, the present study demonstrated that the expression level of gal-3 was significantly upregulated in patients with NPC compared with patients with CR, and that the expression level of gal-3 was associated with the inflammatory state of tumor tissues from patients with NPC. Knockdown of gal-3 in 5-8F and 6-10B cells using gal-3 shRNA led to the inhibition of cell proliferation and migration, promotion 
of cell apoptosis and downregulation of MMP-9 and IL-8 expression levels. Moreover, knockdown of gal-3 suppressed the phosphorylation of ERK1/2 and Akt, which may highlight the underlying mechanism of gal-3 in NPC. Therefore, gal-3 may be a potential therapeutic target for NPC.

\section{Acknowledgements}

Not applicable.

\section{Funding}

This work was supported by the National Natural Science Foundation of China (grant no. 81974112), the National Natural Science Foundation of China (grant no. 81873494), the National Natural Science Foundation of China (grant no. 81922012), the Hunan Natural Science Foundation (grant no. 2018JJ2665) and the Hunan Natural Science Foundation (grant no. 2017JJ2396).

\section{Availability of data and materials}

The datasets used and/or analyzed during the current study are available from the corresponding author on reasonable request.

\section{Authors' contributions}

ML and YBC completed the experiments and wrote the manuscript. FL, JQQ and LCR collected the tissues, conducted the immunohistochemical assay, evaluated the expression levels of gal-3, IL-6, and IL-8 in tissues, and recorded the patients' data. JC and CET designed the study, confirmed the authenticity of all the raw data and helped finalize the manuscript. All authors read and approved the final manuscript.

\section{Ethics approval and consent to participate}

The study was approved by the Ethic Committee of Xiangya Hospital Central South University. Written informed consent was obtained from all patients prior to inclusion into the study.

\section{Patient consent for publication}

Patients agreed with the publication of this study.

\section{Competing interests}

The authors declare that they have no competing interests.

\section{References}

1. Chen YP, Chan ATC, Le QT, Blanchard P, Sun Y and Ma J: Nasopharyngeal carcinoma. Lancet 394: 64-80, 2019.

2. Cao Y: EBV based cancer prevention and therapy in nasopharyngeal carcinoma. NPJ Precis Oncol 1: 10, 2017.

3. Wu M, Li X, Li X and Li G: Signaling transduction network mediated by tumor suppressor/susceptibility genes in NPC. Curr Genomics 10: 216-222, 2009.

4. Chen W, Zheng R, Baade PD, Zhang S, Zeng H, Bray F, Jemal A, Yu XQ and He J: Cancer statistics in China, 2015. CA Cancer J Clin 66: 115-132, 2016.

5. Siegel RL, Miller KD and Jemal A: Cancer statistics, 2016. CA Cancer J Clin 66: 7-30, 2016
6. Kontny U, Franzen S, Behrends U, Bührlen M, Christiansen H, Delecluse H, Eble M, Feuchtinger T, Gademann G, Granzen B, et al: Diagnosis and treatment of nasopharyngeal carcinoma in children and adolescents - recommendations of the GPOH-NPC study group. Klin Padiatr 228: 105-112,2016.

7. Newlaczyl AU and Yu LG: Galectin-3-a jack-of-all-trades in cancer. Cancer Lett 313: 123-128, 2011.

8. Sciacchitano S, Lavra L, Morgante A, Ulivieri A, Magi F, De Francesco GP, Bellotti C, Salehi LB and Ricci A: Galectin-3: One molecule for an alphabet of diseases, from A to Z. Int J Mol Sci 19: 379, 2018.

9. Elad-Sfadia G, Haklai R, Balan E and Kloog Y: Galectin-3 augments K-Ras activation and triggers a Ras signal that attenuates ERK but not phosphoinositide 3-kinase activity. J Biol Chem 279: 34922-34930, 2004.

10. Honjo Y, Nangia-Makker P, Inohara $\mathrm{H}$ and Raz A: Down-regulation of galectin-3 suppresses tumorigenicity of human breast carcinoma cells. Clin Cancer Res 7: 661-668, 2001.

11. Iurisci I, Tinari N, Natoli C, Angelucci D, Cianchetti E and Iacobelli S: Concentrations of galectin-3 in the sera of normal controls and cancer patients. Clin Cancer Res 6: 1389-1393, 2000.

12. Nangia-Makker P, Honjo Y, Sarvis R, Akahani S, Hogan V, Pienta KJ and Raz A: Galectin-3 induces endothelial cell morphogenesis and angiogenesis. Am J Pathol 156: 899-909, 2000.

13. Méndez-Huergo SP, Blidner AG and Rabinovich GA: Galectins: Emerging regulatory checkpoints linking tumor immunity and angiogenesis. Curr Opin Immunol 45: 8-15, 2017.

14. Han YY, Liu K, Xie J, Li F, Wang Y and Yan B: LINC00114 promoted nasopharyngeal carcinoma progression and radioresistance in vitro and in vivo through regulating ERK/JNK signaling pathway via targeting miR-203. Eur Rev Med Pharmacol Sci 24: 2491-2504, 2020.

15. Lv B, Li F, Liu X and Lin L: The tumor-suppressive role of microRNA-873 in nasopharyngeal carcinoma correlates with downregulation of ZIC2 and inhibition of AKT signaling pathway. Cancer Gene Ther 28: 74-88, 2020.

16. Livak KJ and Schmittgen TD: Analysis of relative gene expression data using real-time quantitative PCR and the 2(-Delta Delta C(T)) method. Methods 25: 402-408, 2001.

17. Hara A and Okayasu I: Cyclooxygenase-2 and inducible nitric oxide synthase expression in human astrocytic gliomas: Correlation with angiogenesis and prognostic significance. Acta Neuropathol 108: 43-48, 2004.

18. Chen Y, Liu F, Han F, Lv L, Tang CE, Xie Z and Luo F: Omentin-1 is associated with atrial fibrillation in patients with cardiac valve disease. BMC Cardiovasc Disord 20: 214, 2020.

19. Moloudizargari M, Moradkhani F, Hekmatirad S, Fallah M, Asghari MH and Reiter RJ: Therapeutic targets of cancer drugs: Modulation by melatonin. Life Sci 267: 118934, 2021.

20. Bray F, Ferlay J, Soerjomataram I, Siegel RL, Torre LA and Jemal A: Global cancer statistics 2018: GLOBOCAN estimates of incidence and mortality worldwide for 36 cancers in 185 countries. CA Cancer J Clin 68: 394-424, 2018.

21. Bruce JP, Yip K, Bratman SV, Ito E and Liu FF: Nasopharyngeal cancer: Molecular landscape. J Clin Oncol 33: 3346-3355, 2015.

22. Tang LL, Chen WQ, Xue WQ, He YQ, Zheng RS, Zeng YX and Jia WH: Global trends in incidence and mortality of nasopharyngeal carcinoma. Cancer Lett 374: 22-30, 2016.

23. Lee AW, Ng WT, Chan YH, Sze H, Chan C and Lam TH: The battle against nasopharyngeal cancer. Radiother Oncol 104: 272-278, 2012.

24. Thijssen VL, Heusschen R, Caers J and Griffioen AW: Galectin expression in cancer diagnosis and prognosis: A systematic review. Biochim Biophys Acta 1855: 235-247, 2015.

25. Tang CE, Tan T, Li C, Chen ZC, Ruan L, Wang HH, Su T, Zhang PF and Xiao ZQ: Identification of Galectin-1 as a novel biomarker in nasopharyngeal carcinoma by proteomic analysis. Oncol Rep 24: 495-500, 2010.

26. Wu KL, Kuo CM, Huang EY, Pan HM, Huang CC, Chen YF, Hsiao CC and Yang KD: Extracellular galectin-3 facilitates colon cancer cell migration and is related to the epidermal growth factor receptor. Am J Transl Res 10: 2402-2412, 2018.

27. Wang L, Li YS, Yu LG, Zhang XK, Zhao L, Gong FL, Yang XX and Guo XL: Galectin-3 expression and secretion by tumor-associated macrophages in hypoxia promotes breast cancer progression. Biochem Pharmacol 178: 114113, 2020. 
28. Li CH, Chang YC, Hsiao $\mathrm{M}$ and Liang SM: FOXD1 and Gal-3 form a positive regulatory loop to regulate lung cancer aggressiveness. Cancers (Basel) 11: 1897, 2019.

29. Teymoortash A, Pientka A, Schrader C, Tiemann M and Werner JA: Expression of galectin-3 in adenoid cystic carcinoma of the head and neck and its relationship with distant metastasis. J Cancer Res Clin Oncol 132: 51-56, 2006.

30. Chien MH, Lin CW, Cheng CW, Wen YC and Yang SF: Matrix metalloproteinase-2 as a target for head and neck cancer therapy. Expert Opin Ther Targets 17: 203-216, 2013.

31. Zhang $X$ and Zhuang R: Dione-thiophene conjugate inhibits proliferation and metastasis of nasopharyngeal carcinoma cells through calcium binding protein-P down-regulation. Eur J Med Chem 168: 199-206, 2019.

32. Lan YY, Chang FH, Tsai JH and Chang Y: Epstein-Barr virus Rta promotes invasion of bystander tumor cells through paracrine of matrix metalloproteinase 9. Biochem Biophys Res Commun 503: 2160-2166, 2018

33. Li YY, Chung GT, Lui VW, To KF, Ma BB, Chow C, Woo JK, Yip KY, Seo J, Hui EP, et al: Exome and genome sequencing of nasopharynx cancer identifies NF-kappaB pathway activating mutations. Nat Commun 8: 14121, 2017.

34. Xia Y, Shen S and Verma IM: NF-kappaB, an active player in human cancers. Cancer Immunol Res 2: 823-830, 2014.

35. Zhang X, Yang J, Bian Z, Shi D and Cao Z: Long noncoding RNA DANCR promotes nasopharyngeal carcinoma progression by interacting with STAT3, enhancing IL-6/JAK1/STAT3 signaling. Biomed Pharmacother 113: 108713, 2019.
36. Farnworth SL, Henderson NC, Mackinnon AC, Atkinson KM, Wilkinson T, Dhaliwal K, Hayashi K, Simpson AJ, Rossi AG, Haslett $\mathrm{C}$ and Sethi T: Galectin-3 reduces the severity of pneumococcal pneumonia by augmenting neutrophil function. Am J Pathol 172: 395-405, 2008.

37. Wang L, Friess H, Zhu Z, Frigeri L, Zimmermann A, Korc M, Berberat $\mathrm{PO}$ and Büchler MW: Galectin-1 and galectin-3 in chronic pancreatitis. Lab. Invest 80: 1233-1241, 2000.

38. Li Y, Lv Y, Cheng C, Huang Y, Yang L, He J, Tao X, Hu Y, Ma Y, Su Y, et al: SPEN induces miR-4652-3p to target HIPK2 in nasopharyngeal carcinoma. Cell Death Dis 11: 509, 2020.

39. Zheng P, Chen X, Xie J, Chen X, Lin S, Ye L, Chen L, Lin J, Yu X and Zheng M: Capn4 is induced by and required for Epstein-Barr virus latent membrane protein 1 promotion of nasopharyngeal carcinoma metastasis through ERK/AP-1 signaling. Cancer Sci 111: 72-83, 2020.

40. Wang Z, Liu G, Mao J, Xie M, Zhao M, Guo X, Liang S, Li H, Li X and Wang R: IGF-1R inhibition suppresses cell proliferation and increases radiosensitivity in nasopharyngeal carcinoma cells. Mediators Inflamm 2019: 5497467, 2019.

(i) (3) (9) This work is licensed under a Creative Commons

EY No No Attribution-NonCommercial-NoDerivatives 4.0 International (CC BY-NC-ND 4.0) License. 